\title{
Family planning services in Sri Lanka: Clients' nonmedical expectations and the health systems responsiveness
}

\author{
WL S P Perera ${ }^{1}$, Lillian Mwanri ${ }^{2}$, Rohini De A Seneviratne ${ }^{3}$, Thushara Fernando ${ }^{4}$
}

${ }^{1}$ Post Doctoral Visiting Fellow, ${ }^{2}$ Public Health Physician (FAFPHM) and Course Coordinator, Master of Health and International Development, Discipline of Public Health, School of Medicine, Flinders University, Adelaide, Australia. ${ }^{3}$ Head, Department of Community Medicine, Faculty of Medicine, University of Colombo, Sri Lanka. ${ }^{4}$ WHO Representative to Bangladesh, World Health Organization, Dhaka, Bangladesh.

\section{Abstract}

Nonmedical expectations of the service seekers is an integral component of care that must be given due respect by the healthcare providers in delivering health services. This paper presents findings of a qualitative study conducted exploring the many dimensions of health systems responsiveness (HSR) in relation to family planning (FP) services provided by the government public health sector of Sri Lanka. Clients visiting the government FP clinics in Colombo district were interviewed using a semi structured moderator guide. Interviews covered three core domains of HSR including (i) clinic environment, (ii) ease of access to services and (iii) clients' choice. Content analysis was carried out identifying themes around the domains. In relation to domain 'clinic environment', three subthemes were identified. These included (i) expectations from a 'free service', (ii) disorganization and (iii) sanitary facilities. Similarly, five subthemes emerged under the domain 'ease of access to services', namely, (i) outreach clinics, (ii) domiciliary services, (iii) social networking at MCH clinics, (iv) poly clinic/combined clinic setting and (v) working clients. In relation to the domain 'clients' choice' three subthemes emerged including, (i) service provider selecting the method, (ii) selecting the service provider and (iii) selecting the clinic. The findings emphasize that the healthcare providers must be enlightened on nonmedical aspects of service delivery and HSR must be taken into consideration in delivering FP services.

Keywords: Health system responsiveness, Family planning, Clients' choice, Clinic environment, Ease of access to services, Sri Lanka.

\section{Introduction}

\section{Healthcare system responsiveness}

How health systems responds to legitimate nonmedical expectations of its service seekers was defined as a new concept, named Health Systems Responsiveness (HSR), by the World Health Organization (WHO) in the late 1990s. ${ }^{1-3}$ Described as a multi-domain concept, HSR comprised eight domains, namely, dignity, autonomy, confidentiality, communication, prompt attention, basic amenities, choice of healthcare provider and social support. ${ }^{1}$

The key objective of any health system, is undoubtedly improving the health status of service seekers. ${ }^{1}$ However, it is not the only expectation service seekers may have. Nonmedical aspects of care could be equally or even more important to service seekers over technical aspects of healthcare. ${ }^{4}$ This has been highlighted over the years including current literature on Social Determinants of Health. ${ }^{5}$ Therefore, it is important that health systems are visited time to time with regular assessment of HSR to ensure delivery of effective and efficient services.

\section{Practice Points}

- Nonmedical expectations of the service seekers should be given due respect by the healthcare providers in delivering services.

- Reorganising the physical facilities of the $\mathrm{MCH}$ clinics (including sanitary facilities) to create a comfortable environment is essential to improve the client satisfaction.

- Provision of informed choices and flexibility of services should be extended to the clients in delivering FP services.

- Healthcare provisions at outreach clinics should be strengthened to expand the FP services at the field level.

- Healthcare providers must be enlightened on nonmedical aspects of service delivery and HSR must be taken into consideration in delivering effective FP services.

Correspondence: Dr W L S P Perera, Post Doctoral Visiting Fellow, Discipline of Public Health, School of Medicine, Flinders University, GPO Box 2100, Adelaide 5001, Australia. E-mail: sugi.perera@flinders.edu.au, sugandhikaperera@gmail.com.

South East Asia Journal of Public Health 2012;2(1):54-59. (C) 2012 Perera et al., publisher and licensee Public Health Foundation Bangladesh. This is an Open Access article which permits unrestricted non-commercial use, provided the original work is properly cited. 
Family planning service provision and improvements maternal health

The United Nations (UN) Millennium Declaration in 2000 was an important commitment agreed upon by all governments to create an equally better life for all people. ${ }^{6}$ The Declaration included Millennium Development Goals (MDG) covering eight core areas and setting targets to be achieved by each nation by the year 2015, with the 1990 levels set as benchmarks. ${ }^{6}$ Out of the eight areas identified, three were directly related to the health of people. One of these three was the improvement of maternal health which focused on maternal mortality (Target 5.A) and access to reproductive health (Target 5.B). ${ }^{6}$ Access to reproductive health services has been elaborated with special emphasis on universal access to family planning (FP) services. Though FP services are available globally, inequality of service provision has been identified; leaving the 'unmet need' in FP services a notable problem for many states. ${ }^{6}$

\section{Sri Lankan FP service provision}

Family planning services was introduced to Sri Lanka in the early 1960 s. $^{7-11}$ It was well accepted by many families and today, it has become a critical component of family health services provided by both the government and private sectors. ${ }^{10-11}$ The government is the leading provider which is coordinated by the Family Health Bureau (FHB) of the Ministry of Health ${ }^{9}$. The FHB coordinates services in all parts of the country through the Maternal and Child Health Services, delivered by field public health workers, of whom the Public Health Midwife (PHM) commonly known "miss" is a key stakeholder. ${ }^{10-11}$ The PHM resides in the geographical area assigned to her, named the 'PHM area', with a population of approximately 3000 . She provides all field level $\mathrm{MCH}$ related care, to eligible families of 'her' area. ${ }^{10}$ With regard to FP services, the PHM coordinates the provision of Oral Contraceptive Pill (OCP), condoms, implants, Depomedroxy Progesterone Acetate (DMPA) and Intrauterine Contraceptive Device (IUD) as the modern temporary methods through domiciliary and clinic services.

The Demographic and Health Survey conducted in 2006/7 revealed the Contraceptive Prevalence Rate of Sri Lanka was $68 \%$ with the modern method prevalence being $53 \%{ }^{7}$ The reported rate of unmet need in FP was around $10 \%$ with a wide range from $4-18 \% .^{10-11}$ Gaps in HSR in FP service provision could be a factor contributing to the high unmet need.

\section{Aim}

In 2009, a locally conducted survey determined that the important domains of HSR in relation to FP service provision were (i) being treated with dignity, (ii) clinic environment, (iii) ease of access to services, (iv) clients' choice, (v) confidentiality and (vi) communication. ${ }^{12}$ Though domains were identified, an assessment of HSR of FP services using the domains has not been published internationally in literature. The WHO published results of the World Health Survey conducted in 2002/2003 which included an assessment of HSR. ${ }^{13}$ However, this was a general assessment of the healthcare system which provided little insight to HSR of FP services. The aim of the current paper was to explore perceptions of clients seeking FP services from government public healthcare institutions on domains, clinic environment, ease of access to services and clients' choice.

\section{Materials and methods}

This qualitative study was conducted in government sector FP clinics in Colombo district between May August 2010. Colombo district has been the centre of business and administration from the British era. ${ }^{14}$ Spanning across $697 \mathrm{~km}^{2}$ it is the district with the highest population density of Sri Lanka. ${ }^{14}$ The FP clinics in Colombo which were registered by the FHB were selected for the survey. One interview per clinic was conducted till theoretical saturation was reached. ${ }^{15-17}$ Selection of participants was done through purposive sampling. ${ }^{17}$ Voluntary willingness to participate with consent for tape recording the interviews was obtained from participants following the explanation of the objective of the survey. Confidentiality of information provided was ensured to participants. Ethics approval was obtained from the Ethics Review Committee of the Faculty of Medicine, University of Colombo.

Interviews were conducted and notes taken by the Principal Investigator (SP) outside of clinics. The SP was trained on conducting in-depth interviews by an expert on qualitative research at the Faculty of Medicine, University of Colombo. Discussions were conducted in Sinhala, the local language of most participants. A moderator guide with semi-structured open-ended questions was used. The guide was pretested in a clinic outside of the study area and necessary modifications were made. The discussions were tape recorded, verbal responses written down and nonverbal expressions noted.

Each interview lasted approximately 90 minutes. Interviews were transcribed by SP which was translated to English by a professional translator. The transcripts were read at least twice by three authors and core themes identified and coded. Theoretical saturation was achieved after 38 interviews.

\section{Results}

The basic socio-demographic characteristics and the current use of FP of those who participated in the indepth interviews are given in Table 1.

All participants were married females coming from a wide range of socio-economic backgrounds. Participants aged between 17 to 43 years and included respondents with no formal schooling to university postgraduates. Majority of respondents $(23 / 38 ; 60.5 \%)$ were housewives and all were users of all five modern FP temporary methods provided at the clinic.

Domain 1: Clinic environment

A pleasant physical environment in clinics tends to 
make the visit a pleasant one to the client ${ }^{18}$. Though desired standard of physical facilities largely depends on expectations of respondents and the local context, basic facilities are expected by any service seeker.

Three themes were identified in relation to the domain: (i) expectations from a 'free service', (ii) disorganization and (iii) sanitary facilities.

\section{Expectations from a 'free service'}

Majority felt it was incorrect to expect 'luxuries' from healthcare services which were provided 'free'. Their expectations of a comfortable clinic environment were low, making them satisfied with whatever physical facilities provided.

Table 1: Socio-demographic characteristics of the respondents

\begin{tabular}{|l|c|}
\hline \multicolumn{1}{|c|}{$\begin{array}{c}\text { Socio-demographic } \\
\text { characteristics }\end{array}$} & $\begin{array}{c}\text { Respondents } \\
(\%)\end{array}$ \\
\hline Age (years) & $3(7.9 \%)$ \\
\hline$<20$ & $7(18.4 \%)$ \\
$20-24$ & $9(23.7 \%)$ \\
$30-34$ & $9(23.7 \%)$ \\
$35-39$ & $7(18.4 \%)$ \\
$40-49$ & $3(7.9 \%)$ \\
\hline Education & $29(5.3 \%)$ \\
\hline No schooling & $4(10.5 \%)$ \\
Primary education & $13(34.2 \%)$ \\
General Certificate of Education & $9(23.7 \%)$ \\
(Ordinary Level) & $4(10.5 \%)$ \\
(Advanced Level) & $4(10.5 \%)$ \\
Diploma/ Vocational training & $2(5.3 \%)$ \\
\hline Degree & $15(39.5 \%)$ \\
Post Graduate degree & $23(60.5 \%)$ \\
\hline Employment status & $4(10.5 \%)$ \\
\hline Currently employed & $6(15.8 \%)$ \\
\hline Currently not employed & $10(26.3 \%)$ \\
\hline OCP & $7(18.4 \%)$ \\
Condoms FP method & $(29 \%)$ \\
\hline
\end{tabular}

Keys: OCP- Oral Contraceptive Pill, DMPA- Depomedroxy Progesterone Acetate, IUD- Intrauterine Contraceptive Device.
'We are given quality services free. It is unfair to ask for more facilities. Anyone wanting better facilities can go to the private sector'. ID 2 (39 years)

\section{Disorganization}

Many felt the clinics were 'disorganized' which was sometimes 'uncomfortable'. Some were of view that clients should be brought to the clinic on appointment basis. Rearranging clinics managing space efficiently was suggested as one strategy to improve the environment.

'The clinic is quite disorganized. Earlier we were asked to come on appointments with no overcrowding. Also there are broken furniture occupying spaces unnecessarily and if reorganized it would be more comfortable.' ID 14 (30years)

\section{Sanitary facilities}

Sanitary facilities, particularly toilets, in many clinics seemed to be unhygienic. Participants felt this was not given due interest as most clinic centers had separate toilets only for staff.

'Staff should pay more attention to toilets. There is no running water and you cannot close the door. Toilets are essential as sometimes we have to stay here for over two hours.' ID 10 (17 years)

\section{Domain 2: Ease of access to services}

Ease of access to services covers geographical accessibility, convenience of days and times of the clinics as well as waiting times ${ }^{4,12}$. The following themes were identified during interviews.

Outreach clinics

The MCH clinics are conducted as outreach clinics in all PHM areas around the country making a clinic available to clients within a few kilometers which was walking distance to many.

'I can come here within five minutes on foot. We are fortunate to have services so close to home. ID 17.' (32 years)

\section{Domiciliary services}

Participants commented positively on domiciliary visits made by PHM. The PHM has to visit eligible families regularly to provide FP services and OCP and condoms if required.

'Generally I get all my pills (OCP) from "miss" when she comes home. We can also come to the clinic. This ease of access is what makes me use pills.' ID 21 (31 years)

\section{Social networking at MCH clinics}

Majority of participants were housewives and many considered this an opportunity to socialize with friends and neighbors. Long waiting times was not considered a problem to many. 
'As I do not work, I do not get to go out much. A lot of my neighbors and friends come here for services and I get a chance to chat with them during the waiting time.' ID 27 (36 years)

\section{Poly clinic, combined clinic setting}

Almost all clinics are conducted as poly or combined clinics where antenatal as well as child welfare services are provided. This gave an opportunity for mothers to visit the clinic for all their children's needs and FP needs.

'I came to the clinic to get vaccination for my baby as well as a loop (IUD) for me. It was a great blessing for me as I don't have to find separate time to come for the loop.' ID 36 (27 years)

\section{Working clients}

Clinics were mostly conducted on weekdays in the morning posing an obstacle for working mothers. Some working mothers expressed dissatisfaction on the accessibility to $\mathrm{MCH}$ services of the government sector.

'It is very inconvenient for me to come on a weekday as I have to obtain leave from work. They conduct Saturday morning clinics for those who work, but I work on Saturday too.' ID 5 (28 years)

Domain 3: Clients' choice

Clients' choice is another domain of HSR which includes the choice of the method, choice of the service provider as well as choice of the clinic to obtain FP services. $^{4,12}$

Three themes were identified during discussions which were (i) service provider selecting the method, (ii) selecting the provider and (iii) selecting the clinic.

Service provider selecting the method

It was not surprising to note that many clients were satisfied to give the responsibility of choosing a suitable FP method to the providers.

'I have been using the injection for over five years now and it has not given any trouble. "Miss' has the knowledge of what is good for us.' ID 34 (37 years)

However, there were negative expressions by two respondents about recommending a client of a suitable method. In the local scenario, very little counseling happens and it is mainly the PHM who recommends methods to clients.

'I wanted to use injections after my second baby as I did after my first baby. But "miss" was adamant that I get a loop as I had completed my family. I didn't like it but she kept on pestering me till I got it. Now it is giving me a lot of aches and pains. 'ID 9 (36 years)

Selecting the service provider

In the local setting, little choice is given to clients to select a service provider in clinics. One PHM administers all DMPA while the Medical Officer (MO) or the Public Health Nursing Sister provides implants and loops. Choosing a provider was not expected by many.

'I don't think there is a need to choose service providers as all staff are well trained.' ID 8 (25 years)

Nevertheless, three out of 38 participants, who had come to obtain IUD, stated they would have preferred having a female doctor in the clinic.

'I personally would have preferred if a lady doctor or even the nurse inserted the loop. Since it is females who visit MCH clinics, there must be more female service providers.' ID 32 (29 years)

\section{Selecting the clinic}

The setup was laid for clients from a particular PHM area to always attend the 'area PHM clinic' for FP services which was accepted by many as a good practice. Therefore choosing a clinic for services was not an expectation.

'We always come to "our" clinic for all needs and our "miss" attend to us. I don't think I would choose to go anywhere else. Also our records are in this clinic.' ID 24 (18 years)

\section{Discussion}

Results highlight important issues regarding HSR in the Sri Lankan socio-cultural scenario. Most were satisfactory comments and approval of the manner the health system responds to nonmedical expectations. This is consistent with other surveys conducted locally on patient satisfaction and quality of healthcare services. ${ }^{18-22}$ However, literature also link low-expectations and lack of awareness of patients' rights to high ratings of HSR, especially in developing countries. ${ }^{4}$

A vital reason described in literature in relation to high rating of services and low expectations is the health service being provided 'free'. ${ }^{18-22}$ This was reinforced in the study as many expressed reluctance to expect much comforts in clinics where services were provided 'freeof-charge'. Though services were funded through indirect taxes paid by these service seekers, this was not considered. At most, 'disorganization' was blamed over lack of adequate facilities for discomforts faced. However, respondents were more critical of sanitary facilities. Many clinic centers had separate toilets for staff and hence poor attention was paid on maintenance of facilities for clients. In addition, the clients are generally from the vicinity and therefore, to the service providers, providing these facilities was not a priority as the 'technical service'. ${ }^{18}$

Many respondents expressed the access to services was satisfactory. The government's policy to provide outreach $\mathrm{MCH}$ clinics in all PHM areas provided convenient access to $\mathrm{MCH}$ centers across the country. ${ }^{11}$ The PHM also provided domiciliary services and clients 
on OCP and condoms were able to obtain them at the convenience of their doorstep. ${ }^{18,20}$ When waiting times were discussed, it was found that majority of respondents were housewives not having as much opportunities to go out of their houses and socialize as employed clients. Therefore, even though some had to face longer waiting times, it provided an opportunity to meet neighbors/friends. This was a unique finding not described elsewhere and highlights the socio-cultural context where there is much interaction among neighbors/ friends. The long waiting time could be considered important (in the current study's context) as it aligns with new public health concepts including developing socialnetworking, breaking isolation and building the social capital which have been described as predictors of good health outcomes. ${ }^{23,24}$ Another reason identified was that all $\mathrm{MCH}$ related care could be obtained from the one clinic session. With the poly/combined clinic setting, mothers were able to obtain child health services, e.g. vaccinations, growth monitoring etc. and FP services in one visit. This improved the access to FP services by eligible families.

The negative comment on accessing services highlights the importance of providing services equally to all. The $\mathrm{MCH}$ clinics are conducted on weekdays. Identifying the needs of the working mothers, the FHB has instructed all Medical Officer of Health $(\mathrm{MOH})$ divisions to conduct a 'fortnightly Saturday central clinic' at the $\mathrm{MOH}$ office. The $\mathrm{MOH}$ division is much larger than PHM areas and hence access to this 'central clinic' is not as convenient as outreach clinics in PHM areas making it harder to reach for the working mothers. Also, many working mothers also do work half-day on Saturday morning; the system needs to pay special attention to meet the requirements of these 'employed' clients. In addition, PHM sometimes does not provide enough flexibility to working mothers, may be by serving her early. This is an issue that needs careful consideration which could potentially pose as inequity issue in $\mathrm{MCH}$ service provision and also hinder the country from achieving its MDG targets. ${ }^{24}$

Though the choice provided to clients is an important part of HSR, results show that many do not view being able to choose a method, a provider or a clinic as important. Conventionally, the respondents were quite happy to give the service provider the option of choosing a suitable FP method. Sometimes, there were expressions of dissatisfaction for not being able to select a method when clients were suffering from undesired, side effects. This highlights the importance of counseling and providing adequate information to clients. The DMPA, OCP and condoms are generally provided by the area PHM giving no choice to the clients. Also the respondents did not consider choosing a provider important in the clinic. Only a small proportion (3/38) who had come for IUD insertion which required vaginal examinations expressed the desire to obtain services from a female. This is consistent with the local socio-cultural context where the females are more comfortable being examined by a female, especially when vaginal examinations are required. The local $\mathrm{MCH}$ clinic setup requires the females to mainly visit 'their area PHM clinic' for FP services. This was found to be comfortable setup as respondents were close to their area PHM and the clinic which they visited for all $\mathrm{MCH}$ care. Choosing a clinic and a place for services was not expected.

The study has its own limitations. The inclusion of only one district limits the generalization of findings. Also views of spouses and other relevant informants would enhance further understanding of HSR issues in Sri Lanka. Participants were purposively selected which could lead to possible selection bias. The group with unmet needs in FP, who do not access care despite the services being accessible island wide, the unmarried, divorced and widowed females, who may be having FP needs but are reluctant to visit clinics due to sociocultural reasons, also need to be studied. Therefore, further studies to explore issues of HSR involving all stakeholders and more geographical areas of the country are recommended.

\section{Conclusion}

This study identifies locally relevant information to enhance HSR in FP service delivery in Sri Lanka. Issues identified include improving sanitary facilities in the clinic centers, improving equity of access by working women and training the FP health workers on FP counseling. Addressing nonmedical aspects of care is important in order to provide equitable services and to potentially address the current gap which contributes to the high unmet needs of FP service provision.

\section{References}

1. WHO. World Health Report. Geneva: World Health Organisation, 2000.

2. WHO. Report on WHO meetings of experts: responsiveness concepts and measurement. Geneva: World Health Organization, 2000.

3. WHO. Background paper for the technical consultation on responsiveness concepts and measurement. Geneva: World Health Organisation, 2001.

4. De Silva A. A framework for measuring responsiveness. Geneva: World Health Organization, 2002.

5. WHO. Closing the gap in a generation: health equity through action on the social determinants of health. Geneva: World Health Organization, 2008.

6. United Nations. Millennium Development Goals, Goal 5: improve maternal health. www.un.org/ millenniumgoals/maternal.shtml (accessed March 2012)

7. Ministry of Health care and Nutrition. Demographic and health survey 2006/7. Colombo: Department of Census and Statistics, 2007.

8. Uragoda CG. Medical inscriptions in ancient inscriptions of Sri Lanka. CMJ 1997;22:3-10. 
9. Ministry of Health. Progress report. Colombo: Ministry of Health, 2008.

10. Family Health Bureau. Family health report. Colombo: Family Health Bureau, 2004.

11. Family Health Bureau. Family Health report. Colombo: Family Health Bureau, 2008.

12. Perera WLSP. Health systems responsiveness of family plannig services in colombo district. Colombo: Postgraduate Institute of Medicine, 2011.

13. WHO. World Health Survey. World Health Organisation. www.who.int/whs. (accessed March 2012)

14. Colombo District Secretariat. Introduction to the district of Colombo. http:// www.colombo.dist.gov.lk/ (accessed March 2012)

15. Abramson JH, Abramson ZH. Survey methods in community medicine. London: Churchill Livingston, 1999.

16. Britten N, Fisher B. Qualitative research and general practice. Br J Gen Pract 1993;43: 270-1.

17. Yoddumnern-Attig B, Attig GA, Boonchalaksi W. Qualitative methods or population and health research. Bangkok: Institute for Population and Social Research, 1993.
18. De Silva UG. An assessment of quality of care provided by the FP clinics and field services in DPDHS area Colombo. Colombo: Postgraduate Institute of Medicine, 2004.

19. Tennakoon S. Patient satisfaction survey in a Sri Lankan hospital. Colombo: Postgraduate Institute of Medicine, 1990.

20. Jayatissa KLR. An analysis of utilization, quality and costs of maternal and child health services. Colombo: Postgraduate Institute of Medicine, 1995.

21. Jayasekera HDMH. An assessment of quality of life and satisfaction with care in patients diagnosed with some common cancers. Colombo: Postgraduate Institute of Medicine, 2006.

22. De Silva P. Functional disability, health related quality of life and health care cost profile of young elderly in urban and rural areas of Kalutara district. Colombo: Postgraduate Institute of Medicine, 2010.

23. Baum F. The new public health. $3^{\text {rd }}$ ed. South Melbourne: Oxford University Press, 2008.

24. Wilkinson R, Marmot M. Social determinants of health, the solid facts. $2^{\text {nd }}$ ed. Copenhagen: World Health Organization, 2005. 